\title{
A deglitching circuit for the AA50 D/A converter
}

\author{
JAMES H. HOWARD, JR., DONALD C. BURGY, and JAMES A. BALLAS \\ Catholic University of America, Washington, D.C. 20064
}

\begin{abstract}
Switching transients or "glitches" are frequently encountered in signal processing applications involving digital-to-analog (D/A) conversion. A low-cost "deglitching" circuit is described and implemented for the Digital Equipment Corporation AA50 D/A converter option on the PDP-8/e minicomputer. Switching transients were eliminated using a noninverting, unity-gain sample-and-hold circuit (employing Datel System's SHM-IC-1). Switching transients were reduced to below-noise levels across a wide range of output levels and sampling rates.
\end{abstract}

The use of laboratory digital computers for the synthesis and playback of speech-like and complex nonspeech sounds has become commonplace. In addition, the precise signal control achieved with these methods and the increased availability of digital technology will likely increase their use in the near future. Despite the obvious advantages, users of even the simplest signal processing techniques are likely to encounter a number of problems, the most common being the poor switching or tracking capability of many laboratory digital-to-analog (D/A) converters (Hnatek, 1976). The introduction of switching transients and other singularities or "glitches" into an acoustic signature can lead to audible broad-band distortion at even relatively high signal-to-noise ratios. We have encountered this switching problem in the AA50 12-bit D/A converter on our Digital Equipment Corporation (DEC) PDP-8/e computer, and the present paper describes a low-cost sample-and-hold circuit designed to correct the problem.

The AA50 converter, originally designed for the PDP-8/I, is a 12-bit device (DEC A614 cards) that interfaces to the positive external bus of the PDP-8/e (KA8-E positive I/O bus). Despite its age, the AA50 offers a number of advantages over more recently introduced DEC converters. First, used AA50s are readily available at competitive prices. Second, for an equivalent full-scale range, its 12-bit coding affords a better resolution than the more recent 10-bit offerings (e.g., AA05 and VC8-E). Third, the AA50's logic and electronics are mounted on an extemal 19 in rack and are therefore easily modified for custom applications. Recent DEC converters are mounted on quad-height printed circuit boards that plug into the PDP-8/e omnibus, and they are generally inaccessible for modification.

The authors thank Fred Brandt for his comments on this manuscript, and Dave Talkin for assisting with the FFT analysis. This work was supported by a contract from the Office of Naval Research. Requests for reprints should be addressed to James $H$. Howard, Jr., Department of Psychology, Catholic University of America, Washington, D.C. 20064.
Despite these advantages, the AA50 exhibits poor switching performance across a wide range of common throughput rates $(5-40 \mathrm{kHz})$. Figure 1 displays an oscilloscope trace of an unfiltered signal that illustrates the switching transients or glitches. The spikes vary considerably in amplitude (up to 35\% of peak-to-peak voltage range) and occur within the 25 -microsec settling time of the converter. The problem is not unique to any particular AA50 converter (we have observed it on the output of at least four different A614 cards), and it is probably due to uneven on/off times of the A614's transistor switches. In addition, Hnatek (1976) reports that this problem is frequently encountered in a variety of applications involving high-speed D/A converters, and he recommends that deglitching circuitry be used whenever possible. The broad spectra of these singularitiies clearly include the frequency region within the pass band of an antialiasing filter.

Our strategy in eliminating this problem was to interpose a high-speed sample-and-hold circuit (Chirlian, 1976) on the output of the AA50 that samples the AA50 output during its stable period (i.e., after the output has settled) and holds this level through the unstable switching period. ${ }^{1}$ Since the AA50 logic is

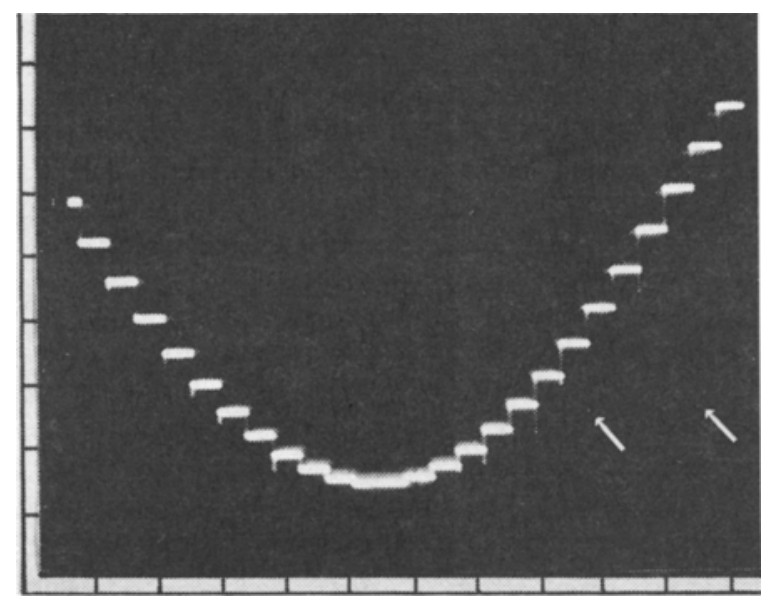

Figure 1. Oscilloscope trace showing switching transients in AA50 output signal. Vertical scale is $2.0 \mathrm{~V} /$ division, and sweep rate is 200 microsec/division. 


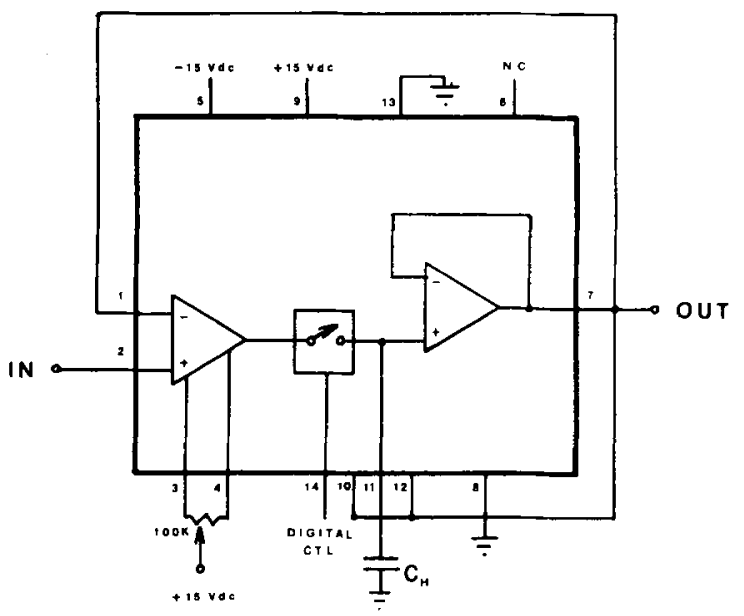

Figure 2. Datel Systems SHM-IC-1 configured for unity-gain noninverting operation. Taken from Datel Systems, Inc. (1978, p. 162). The numbers 1 to 13 indicate pin numbers, and NC indicates no connection.

external to the PDP-8/e, control signals and mounting space are readily available for the sample-and-hold circuit.

\section{DESIGN}

The design is based on Datel System's SHM-IC-1 monolithic integrated-circuit sample-and-hold. Its performance characteristics, economical cost ( $\$ 19$ in singleunit quantities), and convenient packaging (14-pin dual in-line) are well suited to the present application.
Figure 2 displays the SHM-IC-1 configuration for the unity-gain noninverting application we employed. Input to the circuit is applied to the noninverting side of the differential input amplifier. A $100-\mathrm{kohm}$ trimming potentiometer is used to adjust the offset of the input amplifier. A delayed TTL logic signal originating from the AA50 control logic serves as a digital clock input to control the circuit's electronic switch. Switching speed and accuracy as well as voltage droop in the hold mode are determined by the value of the holding capacitor $\left(\mathrm{C}_{\mathrm{H}}\right)$. We achieved satisfactory performance with a .001 -microF polystyrene-type holding capacitor.

The SHM-IC-1 was mounted on a single-height DEC W974 blank module in an unused slot on the AA50 logic rack. The circuit layout and pin connections are displayed in Figure 3. The $+5 \mathrm{~V}_{\mathrm{cc}}$ was taken from the DEC H716 supply used to power the AA50 logic; the $+15 . \mathrm{V} \mathrm{dc}$ and $-15-\mathrm{V}$ dc reference voltages were obtained from the DEC H704 regulated precision power supply. The \pm 10 -V AA50 output is fed through a 1 -kohm trimpot divider circuit before being applied to the input of the sample-and-hold. This feature provides the ability to continuously adjust the full-scale range of the analog output (Brophy, 1977).

The digital control (clock input) for the circuit originates in the strobe pulse (A614, Pin BR2) used by the AA50 logic to load the input buffer of the selected converter. Since this signal initiates a conversion on one of the AA50 output channels, it also indicates the start of the 25-microsec settling period when glitches are likely to occur. Our design includes a delay circuit,

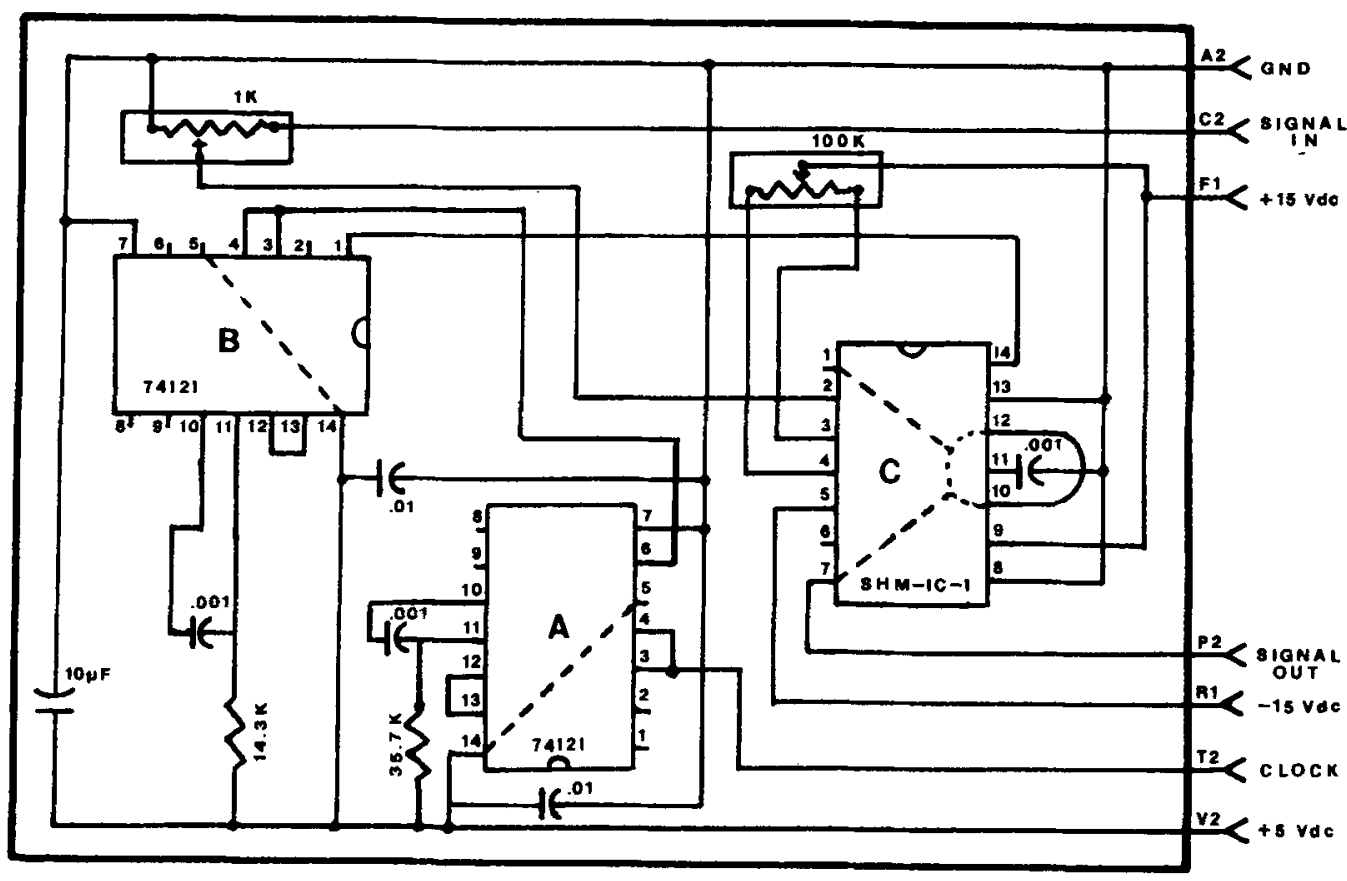

Figure 3. Schematic deglitching circuit layout showing pin connections for the two monostable multivibrators (labeled A and B) and the SHM-IC-1 sample-and-hold (labeled C). Open pins have no connection. 
two SN74121N monostable multivibrators (Texas Instruments, Inc., 1976), to insure that the settling period has passed before the SHM-IC-1 samples the new AA50 output. A timing diagram of this circuit appears in Figure 4. The DEC signal is used as a clock input to trigger a 25 -microsec positive-going pulse in Multivibrator A. The trailing edge of this pulse triggers a 10-microsec negative-going pulse in Multivibrator B. This output is applied to the digital control input of the SHM-IC-1, closing the electronic switch. The sampleand-hold enters its sample mode during this 10 -microsec period and remains in the hold mode at all other times. The 25-microsec delay and 10-microsec sample aperture enable the deglitching circuit to track output rates as high as $28.5 \mathrm{kHz}$. The resistance values necessary to obtain the required delays in the $74121 \mathrm{~s}$ are indicated in Figure 3, and a complete list of circuit components with their approximate costs is presented in Table 1. Assembly, installation, and testing of the circuit requires approximately $10 \mathrm{~h}$.

\section{PERFORMANCE}

Performance specifications of the SHM-IC-1 as configured in Figure 2 are displayed in Table 2. These specifications are for the SHM-IC-1 alone. Ultimate performance of the AA50 with the present sample-andhold circuit will be determined by the specifications of the converter, as well as by the SHM-IC-1. We examined the analog output signal across a wide range of output levels and sampling rates without finding evidence of the switching problem. To further quantify circuit performance, a $20-\mathrm{Hz}$ to $5-\mathrm{kHz}$, narrow-band spectral analysis (FFT algorithm on a PDP-12) was performed on a $200-\mathrm{Hz}$ pure tone (12.5-kHz sampling rate) before and after circuit implementation. A comparison of these spectra revealed an additional 6- to 12-dB attenuation in the harmonic and nonharmonic noise levels across the measured spectrum for the deglitched signal. In conclusion, the circuit described in the present paper provides an economical solution to the problem of

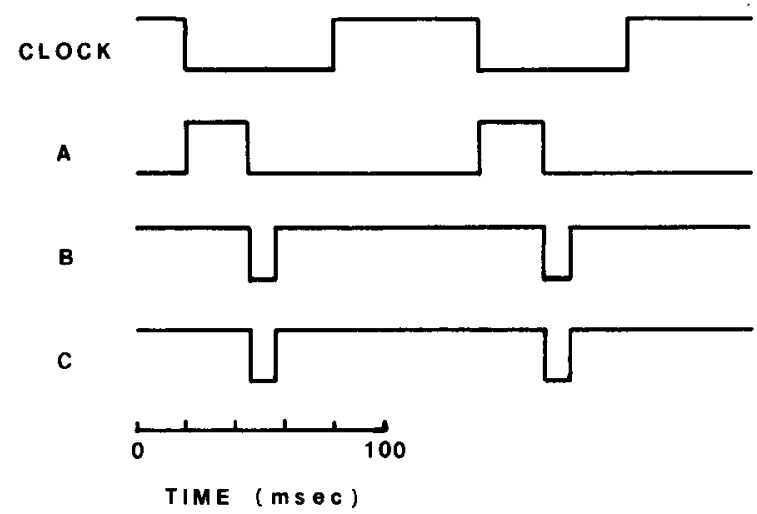

Figure 4. Timing diagram showing relation between AA50 generated logic pulse (clock input), output of the multivibrator delay circuit ( $A$ and $B$ ), and operating mode of the SHM-IC-1 sample-and-hold $(\mathrm{C})$.
Table 1

Circuit Components and Cost

\begin{tabular}{|c|c|c|}
\hline Quantity & Component & Price \\
\hline 1 & Datel Systems SHM-IC-1 & $\$ 19.00$ \\
\hline 1 & $\begin{array}{l}\text { 100-kohm trimming potentiometer } \\
\left(100 \mathrm{ppm} /{ }^{\circ} \mathrm{C} \text { Cermet } 15 \text {-turn type) }\right.\end{array}$ & 3.00 \\
\hline 1 & 1 -kohm trimming potentiometer & .80 \\
\hline 1 & DEC W974 blank module & 9.00 \\
\hline 2 & $\begin{array}{l}\text { SN74121N monostable multi- } \\
\text { vibrators@.40 }\end{array}$ & .80 \\
\hline 3 & $\begin{array}{l}\text { Low-profile 14-pin DIP sockets } \\
@ .20\end{array}$ & .60 \\
\hline 3 & $\begin{array}{l}.001 \text {-microF polystyrene-type } \\
\text { capacitors @.09 }\end{array}$ & .27 \\
\hline 1 & $35.7-\mathrm{kohm}$ resistor $(1 \%$ metal film $)$ & .25 \\
\hline 1 & 14.3-kohm resistor ( $1 \%$ metal film) & .25 \\
\hline 1 & $\begin{array}{l}\text { capacitors@.07 } \\
10 \text {-microF 15-V dc capacitor }\end{array}$ & $\begin{array}{l}.14 \\
.23\end{array}$ \\
\hline $\begin{array}{l}\text { Total } \\
\text { Cost }\end{array}$ & & $\$ 34.34$ \\
\hline
\end{tabular}

Table 2

SHM-IC-1 Specifications as Configured in Figure 2

\begin{tabular}{ll} 
Input Voltage Range & $\pm 10 \mathrm{~V}$ \\
Input Impedance & $10^{8} \mathrm{ohm}$ \\
Output Voltage Range & $\pm 10 \mathrm{~V}$ adjustable \\
Output Current, S.C. protected & $\pm 10 \mathrm{~mA}$ \\
Output Impedance & $.2 \mathrm{ohm}$ \\
Aperture Delay & $50 \mathrm{nsec}$ \\
Aperture Uncertainty & $5 \mathrm{nsec}$ \\
Gain Error, Sampling Mode & $.01 \% \mathrm{max}$ \\
Hold Mode Noise & $350 \mathrm{microV}$ RMS \\
Acquisition Time* & $4 \mathrm{microsec}$ to.1\% \\
& for $10-\mathrm{V} \mathrm{change}$ \\
Tracking Bandwidth* & $2 \mathrm{mHz}$ \\
Hold Mode Droop* & $50 \mathrm{mV} / \mathrm{sec} \max$ \\
\hline
\end{tabular}

*These values reflect SHM-IC-1 performance with a .001-microF holding capacitor and will depend on the value of this capacitor.

switching transients in the output of the AA50 D/A converter.

\section{REFERENCES}

BRophY, J. J. Basic electronics for scientists (3rd ed.). New York: McGraw-Hill, 1977.

Chirlan, P. M. Analysis and design of digital circuits and computer systems. Champaign, Ill: Matrix, 1976.

DAtel Systems, Inc. Datel engineering product handbook. Washington, D.C: Author, 1978.

HNATEK, E. R. A user's handbook of $D / A$ and $A / D$ converters. New York: Wiley, 1976.

Texas Instruments, Inc. The TTL data book for design engineers (2nd ed.). Dallas, Tex: Author, 1976.

\section{NOTE}

1. Alternatively, the A614 converter could be replaced with a state-of-the-art modular D/A converter, such as Datel Model DAC-DG12B. Converters of this type include a selfcontained deglitching circuit and sell for approximately $\$ 250$. 